\title{
APROXIMACIÓN BIOÉTICA A LAS TERAPIAS REPARATIVAS. TRATAMIENTO PARA EL CAMBIO DE LA ORIENTACIÓN HOMOSEXUAL
}

\author{
Gabriel Jaime Montoya Montoya*
}

\begin{abstract}
Resumen: La articulación temática entre bioética y estudios de género resulta evidente, además de vigente, en el análisis de las llamadas terapias reparativas para la "corrección" o cambio de la orientación homosexual. En este artículo se exponen los elementos básicos que, a manera de insumos, permiten el diálogo bioético sobre este asunto. Se exponen los fundamentos de la orientación sexual y su reconocimiento como un derecho humano. Además, se toman algunos elementos teóricos respecto del empleo del poder médico como mecanismo de domesticación. En la parte final se analizan algunas características de las terapias reparativas y se exponen las razones que no hacen éticamente sustentable su aplicación.
\end{abstract}

Palabras clave: homosexualidad, bioética, género, orientación sexual

\section{BIOETHICAL APPROACH TO RE-CONSTRUCTIVE THERAPIES. TREATMENT TO CHANGE THE HOMOSEXUAL ORIENTATION}

\begin{abstract}
The interrelation between bioethics and gender studies is clear and contingent. It is obvious in the analysis of the re-constructive therapies for the "correction" or change of the homosexual orientation. This paper shows the fundamental elements which allow the bioethics dialogue about it in a summary way. Also, the fundamentals of the sexual orientation and their acknowledgement as a human right are exposed. Furthermore, some theoretical aspects are taken with respect to the use of physician power in the domesticating process. The paper finalizes analyzing some characteristics of the re-constructive therapies and showing the ethical arguments which refuse their application
\end{abstract}

Key words: homosexuality, bioethics, gender, sexual orientation

\section{APROXIMAÇÃO BIOÉTICA ÀS TERAPIAS REPARADORAS. TRATAMENTO PARA A MUDANÇA DE ORIENTAÇÃO HOMOSSEXUAL}

Resumo: A articulação temática entre bioética e estudos de gênero resulta evidente, além de vigente, na análise das chamadas terapias reparadoras de "correção" ou mudança da orientação homossexual. Neste artigo se expõem os elementos básicos que, em forma de insumos, permitem o diálogo bioético sobre este assunto. São apresentados os fundamentos da orientação sexual e seu reconhecimento como um direito humano. Além disso, aborda-se alguns elementos teóricos a respeito do emprego do poder médico como mecnismo de domestificação. Na parte final se analizam algumas características das terapias reparadoras e se expõe as razões que não tornam eticamente sustentável sua aplicação.

Palabras chave: homossexualidade, bioética, gênero, orientação sexual

* Médico Psiquiatra. Investigador del Grupo de Investigación en Psiquiatría (Gipsi). Departamento de Psiquiatría, Facultad de Medicina, Universidad de Antioquia. Colombia Correspondencia: gajamon2004@yahoo.com 
"Yo, con el tiempo, he aprendido a disfrutar y a gozarme la homosexualidad.

Pero confieso que no fue fácil, y ahora tampoco es que lo sea, sólo que cuando se acepta y entiende que no es culpa nuestra ser así, ni que es un invento del diablo que se metió en nuestros cuerpos para hacer el mal,

y mucho menos el pecado del que hablan las beatas en misa de cinco, ni que hay que darse golpes en el pecho, ni latigarse, ni siquiera llorar cada noche cuando estamos en nuestras camas bajo las cobijas, tan sólo por ser diferentes al resto de la humanidad. Que tampoco es que sea muy diferente pues,

para ser sinceros, lo que nos diferencia a los gays de los straight no es que nosotros nos acostemos con hombres y ellos no. Ellos

también lo hacen. Lo que nos diferencia, repito, es que nosotros tenemos la corajudez de vivir como nos da la gana, rompiendo todos los esquemas impuestos por la "sociedad" y decidiendo, con la honestidad que esa misma "sociedad" no quiere aceptar, enfrentar todos los paradigmas que dicen que uno se debe casar con una mujer

$y$ tener hijos, y criarlos, y mandarlos al colegio, y producir para darles su mesada mensualmente, y obligarlos a casarse con la mujer que a nosotros, sus padres, nos parezca conveniente, y dejarlos ir un buen día a que vivan lejos de casa para, en ese momento, cuando uno ya tiene cincuenta años, hacer las cosas que siempre quisimos hacer."

“...en Occidente la sexualidad no es lo que callamos, no es lo que estamos obligados a callar, es lo que estamos obligados a confesar. "2

1 Sánchez Baute A. Al diablo la maldita primavera. Bogotá: Alfaguara; 2003.

2 Foucault M. Los anormales. Curso en el College de France (1974-1975). Buenos Aires: Fondo de Cultura Económica; 2001.

\section{Introducción}

La sexualidad ha venido ganando posición en el debate bioético y no es gratuito que tal protagonismo se alcance precisamente por ser una esfera que resulta sustancial y medular a la condición humana. La sociedad ha establecido, a través de las prescripciones morales, los ideales de comportamiento en la vida sexual. La visión tradicional de una pareja hombre-mujer soporta la ineludible condición biológica reproductiva de la sexualidad. No obstante, a partir de la década del sesenta y con la denominada Revolución Sexual, se da una marcada expansión social de las dimensiones placentera de la sexualidad y autoafirmativa de la identidad.

El desarrollo de los métodos de regulación de la fertilidad ofreció la opción de un goce sexual no ligado al temor de un embarazo no deseado. Al mismo tiempo, los grupos minoritarios sexuales - principalmente la comunidad homosexual- alcanzaron mayor visibilidad en la sociedad y canalizaron sus esfuerzos hacia la conquista de unos mínimos en igualdad de trato y oportunidades. Las parejas hombre-hombre y mujer-mujer comenzaron igualmente a aspirar por las condiciones que la sociedad le otorga a las uniones tradicionales. Es en este punto donde surgen algunas tensiones bioéticas cuando, por ejemplo, las parejas de lesbianas solicitan los servicios de la reproducción asistida o las personas transexuales demandan una intervención quirúrgica de reasignación sexual genital.

De alguna manera, se tenía la visión que, con el retiro de la homosexualidad como trastorno psiquiátrico por parte de la Asociación Psiquiátrica Americana (APA), en 1973, lo que seguía era un período de activismo de los movimientos homosexuales que les permitiera alcanzar el reconocimiento legal de las uniones entre parejas de hombres o mujeres, el acceso de las parejas a la seguridad social y, en fin, todos los elementos que evidenciaban la igualdad y la 
no discriminación derivadas de la orientación sexual.

No obstante, surge en Estados Unidos - con más fuerza en los últimos diez años- una creciente corriente conservadora que, además de retirar el reconocimiento de los derechos alcanzados, aboga por la instauración de terapias psicológicas para “corregir" la orientación. Tal movimiento supone, de entrada, que la homosexualidad es un trastorno y que la inclusión de los homosexuales en la sociedad debe proceder mediante su transformación y "curación" por las terapias reparativas.

En este artículo cuestiono los sustentos de tales terapias. Para ello, expongo inicialmente los fundamentos del constructo llamado "orientación sexual"; luego, me refiero al reconocimiento de la orientación sexual como un derecho humano y, enseguida, reviso algunos elementos teóricos respecto del empleo del poder médico como mecanismo de domesticación. En la parte final defino algunas características de las terapias reparativas para, por último, dar algunas razones que hacen éticamente insustentable su aplicación.

\section{Orientación sexual}

Para la OPS/WAS 3 , la orientación sexual es la organización del erotismo y/o apego emocional de un individuo respecto al sexo y género de la pareja implicada en la actividad sexual. Se puede manifestar mediante una combinación de conducta sexual, pensamientos, fantasías o deseos.

Esta definición alude a la multidimensionalidad de la sexualidad humana, que abarca desde el mero contacto sexual corporal hasta su representación psíquica. No obstante, carece

3 Organización Panamericana de la Salud/World Association for Sexual Health. de contenido respecto del entorno social y cultural donde tal orientación sexual es asumida. Aunque se hayan dado significativos avances en la equidad para las minorías sexuales, es un hecho que el panorama mundial es bastante asimétrico en el asunto de la homosexualidad y la bisexualidad.

Por ejemplo, en países como Egipto y Botswana está criminalizada su práctica e incluso las personas van a la cárcel por incurrir en actividades homosexuales(1). En Rusia se contempla la participación obligatoria en terapias psicológicas para remover las conductas homoeróticas de aquellos sorprendidos por la ley. En el otro extremo, los países nórdicos reconocen la igualdad de derechos para las parejas homosexuales e, incluso, permiten la adopción de hijos y el uso de técnicas de reproducción asistida.

De tal suerte que la expresión de la orientación sexual resulta sustancialmente modificada por el entorno social, cultural, político, económico y religioso.

La sexualidad humana se compone de varias esferas. En una primera está el sexo, relacionado principalmente con la condición biológica y anatómica de macho o hembra, los genitales y las características sexuales primarias y secundarias. El género está conectado con esta realidad biológica, pero se construye desde lo social, lo histórico y lo cultural, para dar lo que denominamos "masculino" o "femenino", en tanto roles que asumen o le son asignados a los individuos sexuados. La identidad sexual está representada en la asunción individual de la condición de hombre o mujer, con todas las consecuencias que ello lleva en el orden relacional humano. Otra esfera de la sexualidad humana está configurada por la orientación sexual: en este caso se hace referencia, en esencia, al sexo que estimula psicológica, afectiva y sexualmente a un individuo(2). 
Los heterosexuales responden eróticamente a personas del sexo opuesto, los homosexuales a personas de su mismo sexo y los bisexuales a personas de ambos sexos.

La orientación sexual es multidimensional e integra las facetas de la identidad sexual personal (las características físicas masculinas o femeninas, así como la identificación con un género -masculino o femenino- y con el rol social del mismo) con otras dimensiones de la sexualidad como el comportamiento, las fantasías y los vínculos emocionales(3).

Desde la visión de John Money, investigador americano reconocido por sus trabajos en sexualidad experimental, todos los seres humanos poseen una bisexualidad potencial de varios órdenes: gonadal, cerebral y psicológica. El ser humano presenta finalmente alguna de las orientaciones sexuales en el proceso del desarrollo psicosexual(2). En la actualidad se tiene la visión de que tal proceso no corresponde a un estado inmutable, sino que, más bien, es un proceso de continua interacción entre la estructura biológica y psíquica de la persona y el entorno donde expresa su sexualidad. No obstante, resulta conveniente aclarar que no se ha demostrado que alguna de las orientaciones sexuales obedezca a una elección voluntaria individual. No se ha encontrado que sólo un factor genético, anatómico o conductual dé cuenta completa de toda la orientación sexual.

Desde 1973, la APA retiró la homosexualidad de la lista de enfermedades mentales y adoptó una resolución rechazando la discriminación de las personas homosexuales. En 1987 se excluyó la categoría de homosexualidad egodistónica del Diagnostic and Statistical Manual of Mental Disorders (DSM). En 1993 la Organización Mundial de la Salud (OMS) removió la homosexualidad de la Clasificación Internacional de Enfermedades(4). Desde entonces, se la ha descrito como una condición más del ser humano en lo relacionado con su orientación sexual. Sin embargo, en los últimos años han surgido posiciones, desde la psiquiatría, que defienden una mayor atención a las personas homosexuales, en la medida en que representan un grupo vulnerable para trastornos depresivos, ansiedad y suicidio, sin que se haya establecido una relación de causalidad con su orientación sexual: sólo se ha encontrado asociación.

Ninguna de las orientaciones sexuales es considerada por sí misma como patológica. De tal manera que, cuando se habla de heterosexualidad, homosexualidad o bisexualidad, no se está enunciando una condición que requiera por sí misma tratamiento. Las dificultades que motivan la atención psiquiátrica están dadas, más bien, por la estigmatización y discriminación que pueden experimentar los homosexuales o bisexuales en un medio adverso a su condición.

Hasta el momento, uno de los enfoques teóricos más aceptados para explicar el origen de la orientación sexual es el de la interacción secuencial propuesto por John Money. Según él, se da una interacción entre estructuras cerebrales, factores hormonales y estímulos sociales que, finalmente, terminan expresando la orientación sexual. "Money considera que el niño predispuesto neurohormonalmente a ser homosexual, estaría destinado solamente en el sentido en que posee una mayor vulnerabilidad a diferenciar una orientación homosexual si el medio social o las experiencias personales lo proveen de las circunstancias precisas en el período crítico correspondiente"(2).

Investigaciones realizadas por grupos alemanes, dirigidos por Dörner, han dado significativo peso al efecto del ambiente hormonal materno en la determinación de la orientación sexual(3). Esto se produciría a través de duplas de la masculinización (mayor influencia de andrógenos)/desmasculinización o la feminización/ 
desfeminización cerebral. Reduciéndolo a términos sencillos, al parecer se daría un patrón de impregnación hormonal más de tipo femenino (baja masculinización y alta feminización) en el cerebro de los fetos de hombres homosexuales y en sentido inverso para los fetos de mujeres lesbianas. No obstante, hay que guardar siempre la cautela antes de extrapolar conclusiones, ya que se trata de aproximaciones teóricas que todavía requieren más comprobación experimental.

\section{Aspectos demográficos y poblacionales}

Se estima que entre el 4 y el $10 \%$ de la población es homosexual y que entre el 15 y $20 \%$ puede haber tenido alguna modalidad (pensamientos, deseos, actos sexuales) de bisexualidad. El resto de la población tendría una orientación heterosexual. Para Kinsey, la población heterosexual exclusiva es de 75 a $81 \%$, mientras que la con orientación sexual que incluye la homosexual (homosexuales más bisexuales) es de 19 a $25 \%$. Por su parte, la población con orientación homosexual exclusiva es de 4 a $6 \%$. Kinsey describió que el $37 \%$ de los hombres americanos había tenido alguna experiencia homosexual con orgasmo, mientras que el 13\% de los hombres, predominantemente heterosexuales, había sentido alguna vez atracción sexual por otro hombre sin expresarlo abiertamente.

La observación de la conducta sexual humana y los resultados de trabajos clásicos, como los de Kinsey, han sugerido que la orientación sexual humana se distribuye a lo largo de un continuum, algo así como una escala de grises que va del blanco al negro. Las polaridades de tal distribución vendrían dadas por la heterosexualidad y la homosexualidad. Las personas se distribuyen en diversos puntos de esta escala. Kinsey propuso una que va desde 0 -con una conducta exclusivamente heterosexual- hasta 6 , con una conducta de predominio homosexual. La bisexualidad se ubicaría en puntajes cerca- nos a 3. Esta visión teórica explica, en parte, lo complejo de determinar, en un momento dado, cuántas personas son homosexuales, bisexuales y heterosexuales en una sociedad, ya que depende de la autoidentificación que ellas hacen de su ubicación en el espectro de orientación sexual y del grado de aceptación que tienen de la misma.

Lo interesante para esta discusión teórica es comprender que la orientación sexual humana corresponde a un conjunto de posiciones variables para la población, pero que no son excluyentes unas de otras. Así, la heterosexualidad es una más de las orientaciones sexuales, al igual que la bisexualidad y la homosexualidad. Ninguna tiene más valor que la otra, todas pesan por igual en la diversidad sexual humana. Para los términos de este trabajo, cuando se habla de persona heterosexual, homosexual o bisexual se hace referencia al patrón predominante en su orientación sexual.

La divulgación y aplicación de la Declaración Universal de los Derechos Humanos y de los Derechos Sexuales y Reproductivos ha buscado que grupos minoritarios puedan contar, cada vez más, con las garantías mínimas para vivir en igualdad de condiciones en la sociedad. No obstante, el panorama mundial suele ser bastante heterogéneo y todavía es evidente la discriminación asociada a la orientaciones homosexual y bisexual.

Para autores como Simon Lewin e Ilan Meyer, la relación entre derechos humanos y salud de las personas gays, lesbianas, bisexuales y transexuales (GLBT) sigue siendo problemática(4). La primera mención a la orientación sexual en un tratado de derechos humanos se dio como parte de la implementación de la Convención Internacional de Derechos Económicos, Sociales y Culturales, en lo referente a las obligaciones de salud de los Estados. Por su parte el Comité de Derechos 
Humanos de Naciones Unidas ha afirmado que la discriminación basada en la orientación sexual viola los estándares internacionales de derechos humanos. Entidades como Amnistía Internacional ubican los problemas de la población GLBT en el discurso internacional de derechos humanos y abogan por su condición como sujetos de derecho desde su particular condición de orientación sexual. En su reporte "Crímenes de odio, conspiración de silencio", esta entidad muestra que la expresión de sexualidades no-heterosexuales tiene mundialmente experiencias similares de estigmatización, deshumanización, discriminación, tortura y tratamiento impuesto, basado en la concepción de que se trata de una enfermedad(6). Todavía hay riesgo de ser estigmatizado e, incluso, de ser incluido forzosamente en un tratamiento psiquiátrico, hecho que sigue sucediendo en algunos países como Rusia y Ukrania. Señalan Lewin y Meyer: "no sorprende que las personas gays, lesbianas, bisexuales y transexuales puedan estar temerosas de revelar su orientación sexual al trabajador de la salud ante el temor de una ruptura de la confidencialidad, abuso o represalias"(5).

La doctrina de los derechos humanos deriva de los ideales de la Revolución Francesa: libertad, igualdad y fraternidad. En este mismo orden se han dado las tres generaciones de Derechos Humanos ${ }^{4}$.

\section{- Derechos Humanos de Primera Genera-} ción (Libertad): apuntan a los derechos fundamentales del ciudadano como la libre asociación, el derecho de propiedad, el de información y el derecho al sufragio.

4 Notas del curso "Bioética y Derecho", dictado por la profesora Ángela Vivanco. Programa Internacional de Formación Ética de la Investigación Biomédica y Psicosocial. Año lectivo 2004. Centro Interdisciplinario de Estudios en Bioética, Universidad de Chile.
- Derechos Humanos de Segunda Generación (Igualdad): buscan corregir las deficiencias de los de primera generación, enfatizan en aspectos como la igualdad de trato ante la ley, el derecho a la educación, la libertad religiosa y el derecho a la salud y a la seguridad social.

- Derechos Humanos de Tercera Generación (Fraternidad): se dividen en derechos de primer plano -derechos de consumidores y pacientes, derecho a un ambiente sano, a la paz, al desarrollo- y de segundo plano -derivaciones jurídicas que realzan la libertad y la igualdad y con esto dan entrada a los Derechos Sexuales y Reproductivos.

Los Derechos Sexuales y Reproductivos, que incluyen el respeto a la diversidad sexual, se fundamentan en dos premisas: la igualdad traducida en no discriminación arbitraria y la autodeterminación de los sujetos, conectada con su autonomía y sustentada por la condición inalienable de la dignidad del ser humano. La primera comprende la no discriminación con base en la identidad y la opción sexual, de manera que la persona tenga igualdad para acceder a los recursos y oportunidades, al igual que el resto de los miembros de la sociedad. La segunda alude al derecho a una vida sexual placentera, a tener una opción sexual y a lo que podríamos denominar autocomponerse, según la perspectiva teórica de Maturana y Varela.

Para hacer la interpretación de estos elementos se debe considerar las condiciones de cada sociedad y su nivel particular de elaboración acerca del respeto a la diferencia en lo sexual. El proceso parte con eliminar la discriminación; sigue con la igualdad de trato, comprendida en el concepto de equidad, y se integra finalmente en la "sociedad de la diferencia".

Los Derechos Sexuales y Reproductivos cobraron protagonismo desde 1993 en el marco 
de la Conferencia Mundial de Derechos Humanos, en Viena, de la que emergió la Declaración y Programa de Acción para la eliminación de la violencia basada en el género, el acoso y la explotación sexual(7). Más adelante, la Conferencia Internacional de Población y Desarrollo de El Cairo, en 1994, introdujo el concepto de "salud sexual" en el registro positivo, como una de las experiencias humanas que debe ser cuidada y fomentada.

Como efecto de la acción ejercida por la Asociación Internacional de Gays y Lesbianas (ILGA), fundada en 1978, la Corte Europea de Derechos Humanos decidió que la criminalización de actos homosexuales mutuamente consentidos contravenía el derecho a la privacidad. Sudáfrica es el único país que garantiza explícitamente en su Constitución, adoptada en 1996, la protección de las personas homosexuales contra cualquier forma de discriminación(8).

\section{La visión teórica de Foucault del "individuo a corregir"}

En su libro "Los anormales", Michel Foucault(9) explora las relaciones entre saber médico psiquiátrico y poder social. Toma el entorno de los siglos XVIII y XIX para enfocarse en aquellos individuos "peligrosos" a los que la misma sociedad denomina "anormales". Se trata esencialmente de tres grupos: en el primero, que corresponde al monstruo humano, están incluidos los casos de quimeras y hermafroditas; en el segundo, los individuos a corregir que escapan a la domesticación del cuerpo, del comportamiento y de las actitudes. En el último grupo se ubican los onanistas que reflejan las nuevas relaciones entre la sexualidad y la infancia.

“A partir del momento en que la psiquiatría se plantea efectivamente como tecnología de lo anormal, de los estados anormales fijados here- ditariamente por la genealogía del individuo, el proyecto mismo de curar no tiene sentido. En efecto, lo que desaparece con el contenido patológico del dominio cubierto por la psiquiatría es el sentido terapéutico. La psiquiatría ya no trata de curar, o ya no lo hace en esencia. Puede proponerse (y es lo que se produjo, efectivamente en esa época) funcionar sólo como protección de la sociedad contra los peligros definitivos de que puede ser víctima por parte de gente que se encuentra en un estado anormal. A partir de la medicalización de lo anormal, a partir de la puesta a un lado de lo enfermizo y, por lo tanto, de lo terapéutico, la psiquiatría va a poder atribuirse efectivamente una función que será sólo de protección y de orden.”(9)

Con el incremento del poder del discurso médico, y del psiquiátrico en particular, la sexualidad también se introduce en la visión de lo patológico. Lo hace desde la dimensión física, asunto planteado en la ambigüedad genital y el hermafroditismo, hasta la dimensión conductual, donde las parafilias y la sodomía ocupan la atención de los psiquiatras. La permanencia de esta visión atávica de una psiquiatría protectora de la sociedad frente a la amenaza de los enfermos sexuales (para el caso los homosexuales) explicaría en parte el sustento de las terapias reparativas.

En el siglo XVIII los homosexuales y los bisexuales se clasificaban dentro de lo que Foucault denomina "individuos a corregir". (Este) "es un fenómeno corriente. Tan corriente que presenta -y esa es su primera paradoja- la característica de ser, en cierto modo, regular en su irregularidad". Foucault distingue dos consecuencias fundamentales de este hecho: por un lado, su vecindad con la regla o norma de la sociedad hace que sea difícil distinguirlo o determinarlo como anormal. Por otro, el individuo es incorregible en la medida en que todos los esfuerzos tendientes a modificarlo han fracasado; “...el incorregible, en la medida en 
que lo es, exige en torno de sí cierta cantidad de intervenciones específicas, de sobreintervenciones con respecto a las técnicas conocidas o familiares de domesticación y corrección, es decir, una nueva tecnología de recuperación o sobrecorrección".

$\mathrm{Y}$ de eso se trata precisamente cuando se plantea, en este siglo XXI, la aplicación de las terapias reparativas. Doscientos años atrás la sociedad intentó modificar todo lo que era modificable según su técnica; ahora, con un optimismo científico, intenta incidir donde no ha tenido los resultados esperados: la orientación homosexual. Las terapias reparativas representan los esfuerzos de una nueva domesticación y de una sobreintervención, para emplear el término de Foucault. Sus objetivos no son la comprensión y tolerancia del individuo en su situación particular, y su inclusión integral en la sociedad, sino, más bien, el tratamiento arrasador (la sobreintervención); no atienden a las múltiples condiciones que causan malestar al individuo en su relación con el entorno, sino que depositan de inmediato toda responsabilidad en él y le fabrican en la terapia una solución absoluta, permanente y necesaria para su forzosa inclusión en el mundo de los normales.

\section{Terapias de conversión o terapias reparativas}

El término de terapia reparativa fue acuñado por Joseph Nicolsi(10), en 1991, para hacer referencia a una modalidad psicoterapéutica enfocada a cambiar la orientación homosexual por la heterosexual.

Aunque la homosexualidad no figura en las clasificaciones internacionales de enfermedades, no significa necesariamente que toda la comunidad psiquiátrica acepte tal determinación y que se hayan extinguido los debates sobre el estatuto de la homosexualidad. Al respecto, J. Drescher(11) propone estudiar el asunto desde dos perspectivas:

- Modelo de Normalidad/Identidad: afirma que la homosexualidad es una variación normal de la expresión humana, similar, por ejemplo, a la zurdera; además, incluye la aceptación de la orientación homosexual como integrante de la identidad personal. Acorde con este modelo, las personas homosexuales son vistas como pertenecientes a una minoría que requiere protección de las prácticas discriminatorias y de las creencias de la sociedad mayoritariamente heterosexual. Incluso pueden recibir psicoterapia que facilite la asimilación de la situación actual y canalice la integración de la identidad sexual individual.

- Modelo de Enfermedad/Conducta: sostiene que "la enfermedad o la inmoralidad no pueden proporcionar fundamento para la producción de una identidad normal"(4) $\mathrm{y}$ que las personas que tienen conductas homosexuales no ameritan ser protegidas legalmente como una minoría, ya que, al ser enfermos, lo que deben recibir es un tratamiento. Este modelo es el que da sustento a las terapias reparativas o de "conversión", que buscan "reparar" el daño producido en el desarrollo psicosexual "cambiando" la orientación sexual desenfocada.

Luego de la decisión de la APA, en 1973, el modelo de Enfermedad/Conducta fue cada vez menos citado en la literatura científica. No obstante, permaneció en boca de los sectores más conservadores de la sociedad. Lo que sucedió entonces fue que cambió el ámbito de la discusión: se abandonó el terreno científicomédico y se dio especial énfasis a lo político, donde todavía se sustentaba la visión de los homosexuales como anormales. Allí es donde se bloquean las iniciativas que abogan por la 
igualdad de trato en asuntos como el matrimonio, la adopción de hijos y la seguridad social de las parejas gays y lesbianas.

No obstante, quedaba aún un último bastión de este modelo Enfermedad/Conducta en las teorías psicológicas. Para Freud, la conducta homosexual no era una enfermedad, pero sí una detención o regresión del desarrollo psicosexual. Valiéndose de esta conclusión, Sandor Rado, entre los años 60 y 70, concluye que la única salida no patológica para la orientación sexual es la heterosexualidad y que la homosexualidad requiere un tratamiento, desde la perspectiva psicoanalítica, enfocado a superar esta tergiversación del desarrollo psicosexual.

Se produjo entonces una dualidad en el terreno de la psicoterapia: por un lado, se tenía a la homosexualidad como una condición más de la diversidad sexual, cuyas dificultades radicaban en la inserción con equidad en el entramado social; por otro, se trabajaba en desarrollar una terapia para un trastorno del desarrollo. En este último enfoque se evidencia el acercamiento psicoterapéutico al "individuo a corregir", enunciado por Foucault: la persona homosexual viola la norma de la universalidad de la heterosexualidad e, incluso, amenaza la estructura valórica sexual. La psiquiatría debería actuar entonces y ofrecer una medida que sirva para proteger a la sociedad, como lo hizo en su momento con los "locos" y los delincuentes. La inclusión social de la persona homosexual o bisexual estaría supeditada a la autoaceptación de su estatuto de enfermo, frente al cual se restituye, en plenitud, la soberanía del poder médico. No se puede ser diferente en esta sociedad pluralista y supuestamente posmoderna.

Si bien estos puntos de vista se sostenían con las lecturas axiológicas conservadoras, no contaban con el respaldo que el paradigma científico podía aportar. La plataforma seudo científica que soporta las terapias reparativas se deforma por los pobres resultados obtenidos. Puede ser que las personas consulten porque experimentan dificultades en la expresión social de su orientación -lo que viene apareado, con frecuencia, a cogniciones de culpa-, más no necesariamente por la naturaleza de su deseo erótico-afectivo ni por la orientación que han descubierto en su interior.

Sin embargo, el psicoterapeuta que aplica la terapia reparativa transforma el motivo de la consulta en una queja por la condición sexual $\mathrm{y}$, obedeciendo a un modelo médico del siglo XVIII y XIX, retira lo que considera está malo, así como se extirpa un tumor o se extrae una muela cuando duele. El terapeuta toma partido por su paciente, decide por él, basado en un paternalismo médico que suprime el ejercicio de una autonomía construida con el conocimiento. Se asume, erróneamente, que en todos los homosexuales está latente una heterosexualidad y que son infelices por su condición.

El asunto concreto es que la evidencia de resultados exitosos de la terapia hasta el momento es deficiente. Además, tampoco se cuenta con un modelo patológico y terapéutico lo suficientemente fuerte como para soportar la fuerza de la evidencia clínica. Al respecto la APA, en uno de sus documentos oficiales, expresa: "Como principio general, un terapeuta no debería determinar la meta de un tratamiento de manera coercitiva o a través de influencia encubierta. Las modalidades psicoterapéuticas destinadas a cambiar o 'reparar' la homosexualidad están basadas en teorías del desarrollo cuya validez científica es cuestionable. Más aun, los reportes anecdóticos de 'curas' están contrapuestos por reclamos de daño psicológico. En las últimas cuatro décadas, los terapeutas 'reparativos' no han producido alguna investigación científicamente rigurosa por sustentar sus afirmaciones 
de 'cura'. Hasta que tal investigación esté disponible, la APA recomienda que los médicos éticos se abstengan de cambiar la orientación sexual del individuo, teniendo en mente el dictado médico de primero no hacer daño"(4).

Las terapias reparativas desconocen los avances en la explicación de la orientación sexual, como el propuesto por J. Money. Consideran principalmente que la homosexualidad es un trastorno donde hay una detención y distorsión de desarrollo sexual, que fluye hacia un objetivo errático, búsqueda de placer y no de reproducción. Además, involucran elementos cognitivo-conductuales para afirmar que la persona homosexual refuerza con su conducta un esquema distorsionado de deseo sexual y que los movimientos activistas gay estarían tratando de imponer a la sociedad como normal una conducta aberrante. Las estrategias que emplean son variadas, incluyen desde el condicionamiento aversivo (con choque eléctricos o agentes eméticos) hasta el celibato con reforzamiento de las estructuras mentales de culpa-daño(11).

La mayoría de las terapias reparativas fallan en distinguir la orientación homosexual de las conductas homosexuales. En algunos casos, proclaman que hubo éxito cuando la persona simplemente entró en celibato, pese a que, internamente, su orientación homosexual permanezca intacta; o cuando aumentó o inició los contactos heterosexuales. En este último caso les resulta más aceptable una conducta bisexual con una estructura mental homosexual inhibida.

Los efectos secundarios o colaterales de estas terapias son: empeoramiento de la homofobia internalizada existente, interrupción del desarrollo de una identidad homosexual integrada, empeoramiento de síntomas de depresión y ansiedad, incremento del autorrechazo, baja autoestima, conductas autodestructivas, desarrollo de hostilidad en las relaciones heterosexuales y desconfianza con los profesionales de la salud mental(11).

\section{Aproximación bioética}

Las terapias reparativas llevan implícita la renuncia individual a los derechos sexuales y reproductivos, que apuntan al disfrute pleno de la sexualidad en todas sus modalidades siempre y cuando no esté comprometiendo la integridad y bienestar de terceros. Citando a Diego Gracia: "los temas de la ética de la sexualidad pueden ser enfocados desde esta perspectiva (la ética de la responsabilidad). Hay un canon que es, como ya sabemos, el respeto a los seres humanos. Este respeto se concreta, en el orden privado, en los principios éticos de autonomía y beneficencia. Y como el ejercicio de la sexualidad es básicamente privado, resulta que un acto será moralmente reprobable cuando viole la autonomía de las personas o éstas no lo consideren beneficioso para ellas. Naturalmente, este ejercicio privado y autónomo de la sexualidad tiene unos límites, que vienen marcados por los principios de no-maleficencia y de justicia. Por maleficentes o por injustas, consideramos inmorales todas aquellas conductas que agreden, escandalizan, etc., a personas determinadas, sobre todo si son menores de edad o incapaces. La violación, la agresión sexual, el acoso sexual, el exhibicionismo y la provocación sexual, etc., son conductas moralmente negativas precisamente por eso"(12).

La homosexualidad o la bisexualidad per se no representan una condición que éticamente atente contra el respeto a los seres humanos. El psiquiatra puede evaluar lo moralmente sancionable de las conductas sexuales basado, en principio, en lo maleficente o injusto de las mismas en tanto afectan la integridad del otro y no necesariamente por la orientación sexual de quien las realiza. 
Más adelante, Gracia afirma: "el ejercicio de la sexualidad pertenece a la esfera de lo privado, más aún de lo íntimo, $\mathrm{y}$, por tanto, está protegido por el derecho a la privacidad y a la intimidad (...) el respeto a la diversidad sexual y al ejercicio libre de la sexualidad, por supuesto dentro de ciertos límites que las sociedades tienen que establecer por consenso, es un deber perfecto, generalmente conocido con los nombres de derecho a la intimidad y a la privacidad".

Así vista, la orientación sexual, como expresión de la diversidad sexual, debe estar protegida por el carácter de intimidad y privacidad que le acompañan en su esencia. Al introducir las terapias reparativas se está vulnerando precisamente ese derecho a la intimidad y a la diversidad de opciones de desarrollo vital.

Los principales cuestionamientos éticos realizados a las terapias reparativas pueden ser resumidos en lo siguiente:

- No hay suficiente cuerpo de conocimientos, científicamente validados, como para considerar que las terapias reparativas sean efectivas. No puede ser éticamente sustentable presentar o proponer a las personas una terapia que no tiene soporte técnico.

- El proceso de consentimiento informado en las terapias reparativas se distorsiona en la medida en que se magnifican las expectativas de éxito y se aminoran las del fracaso.

- Las terapias reparativas parten de la premisa de que la conducta y la identidad homosexuales son patológicas y, en consecuencia, moralmente inaceptables. Con lo que desconocen el ejercicio responsable de una homosexualidad basada en el respecto a la autonomía y a la dignidad de las personas.
- Si las terapias no tienen el debido soporte científico y técnico, es difícil referir que haya personas competentes para realizarlas. No es ético aplicar procedimientos o técnicas psicoterapéuticas cuando no se cuenta con la idoneidad profesional.

- El empleo de técnicas de disuasión para convencer a las personas homosexuales de que su orientación es patológica o inferior es cuestionable desde las mismas bases de la ética profesional; también lo es la imposición de criterios individuales, derivados de corrientes políticas o religiosas, en la prestación de un servicio de salud mental.

- Las terapias reparativas no son inocuas: con frecuencia exacerban los elementos homofóbicos internalizados con deterioro de la autoestima e incremento del riesgo de suicidio, lo que constituye un inadecuado equilibrio entre los riesgos y los beneficios.

- Algunos centros llegan a cobrar hasta 10.000 dólares por un tratamiento que dura mínimo dos años, sin que se pueda demostrar la efectividad de la terapia aplicada, lo que representa una forma de explotación económica.

Para concluir, las terapias reparativas representan una distorsión del abordaje psicoterapéutico de la diversidad sexual humana. Desconocen los alcances logrados con los Derechos Humanos y, en especial, con los Derechos Sexuales y Reproductivos. Pueden reflejar la tergiversación del poder médicopsiquiátrico y su inclusión en procesos de domesticación y ocultamiento de la diversidad sexual humana. También son éticamente insustentables, al carecer del soporte científico que demuestre sus indicaciones y la efectividad de su intervención. 


\section{Agradecimientos}

El autor desarrolló la revisión temática y la construcción conceptual de este artículo durante su formación en el Programa Internacional en Ética de la Investigación Biomédica y Psicosocial del Centro Interdisciplinario de Estudios en Bioética de la Universidad de Chile. Recibió beca de la Fogarty Foundation, de los NIH de Estados Unidos, Program Training Grant 1R25TW06056-01.

\section{Referencias}

1. Ehleres VJ, Zuyderduin A, Oosthuizen MJ. The well-being of gays, lesbians and bisexuals in Botswana. Issues and innovations in Nursing Practice 2001; 35: 848-856.

2. Giraldo O. Nuestras sexualidades. Cali: Litocencoa; 2002.

3. Coleman E, Tietje L, Harrison J. Homosexuality. En: Reich WT, (ed.) Encyclopedia of Bioethics. Vol. 1. New York: Macmillan; 1995: 1143-1156.

4. American Psychiatric Association. Official actions. Position Statement on Therapies Focused on Attempts to Change Sexual Orientation (Reparative or Conversion Therapies). Am J Psychiatry 2000; 157: 10.

5. Lewin S, Meyer I. Torture and ill-treatment based on sexual identity: the roles and responsibilities of health professionals and their institutions. Health and Human Rights 2002; 6(1).

6. Amnesty International. Crimes of Hate, Conspiracy of silence. Torture and Ill-treatment Based on sexual Identity. London: Amnesty International; 2001.

7. Parker RG. Sexual Rights: Concepts and action. Health and Human Rights 1996; 2(3).

8. Altman D. HIV, homophobia and Human Rights. Health and Human Rights 1996; 2 (4).

9. Foucault M. Los Anormales. Curso en el College de France (1974-1975). Buenos Aires: Fondo de Cultura Económica; 2001.

10. Halpert SC. If it Ain't Broke, Don't fix it: Ethical Considerations Regarding Conversion Therapies. International Journal of Sexuality and Gender Studies 2000; 5(1).

11. Drescher J. Ethical issues in treating gay and lesbian patients. Psychiatr Clin N Am 2002; 25: 605621.

12. Gracia D. Ética de los confines de la vida. En: Ética y vida. Estudios de Bioética 3. Santafé de Bogotá: El Búho; 1998.

Recibido el 23 de agosto de 2006.

Aceptado el 27 de septiembre de 2006. 